Proceedings of the ASME Turbo Expo 2018:

Turbomachinery Technical Conference \& Exposition

June 11-15, 2018, Oslo, Norway

GT2018-75982

\title{
SCALE-RESOLVING SIMULATIONS OF LOW-PRESSURE TURBINE CASCADES WITH WALL ROUGHNESS USING A SPECTRAL-ELEMENT METHOD
}

\author{
Anirban Garai \\ Laslo T. Diosady \\ Science and Technology Corporation \\ Hampton, VA, USA
}

\author{
Scott M. Murman \\ Nateri K. Madavan \\ NASA Ames Research Center \\ Moffett Field, CA, USA
}

\begin{abstract}
The accurate prediction of wall-roughness effects in turbomachinery is becoming critical as turbine designers address airfoil surface quality and degradation concerns arising from the shift to advanced ceramic matrix composite (CMC) or additively-manufactured airfoils operating in higher temperature environments. In this paper, a recently developed computational capability for accurate and efficient scale-resolving simulations of turbomachinery is extended to analyze the boundarylayer separation and transition characteristics in a rough-wall low-pressure turbine (LPT) cascade. The computational capability is based on an entropy-stable discontinuous-Galerkin spectral-element approach that extends to arbitrarily high orders of spatial and temporal accuracy, and is implemented in an efficient manner for a modern high performance computer architecture. Results from the scale-resolving simulations of both smooth and rough airfoil cascades are presented and compared to previous experiments and numerical simulations. The results show that the suction surface boundary layer undergoes laminar separation, transition, and turbulent reattachment for the smooth airfoil cascade, while in the presence of roughness the separation and transition behavior of the suction surface boundary layer is substantially modified. The differences between the smooth and rough airfoil cascades are then highlighted by a detailed analysis of their respective turbulent flow fields.
\end{abstract}

\section{NOMENCLATURE}

$C \quad$ Axial chord length
$C_{f} \quad$ Skin friction coefficient

$D \quad$ Dissipation

$M \quad$ Mass flux variation term

$M a \quad$ Mach number

$M C \quad$ Mean convection term

$P \quad$ Production term

Ps $\quad$ Pressure-strain term

$P t \quad$ Pressure transport term

$Q \quad$ Normalized heat flux

$R_{a} \quad$ Centerline average roughness

Re Reynolds number

Tt Turbulent transport

$\mathrm{Tu} \quad$ Turbulent intensity

$U_{s} \quad$ Mean tangential velocity

$V t \quad$ Viscous transport

$k_{s} \quad$ Equivalent sandgrain roughness height

$n \quad$ Wall normal direction

$p \quad$ Pressure

$p t \quad$ Total pressure

$s \quad$ Surface distance

$t \quad$ Normalized time

$u \quad$ Velocity

$x \quad$ Streamwise coordinate direction

$y \quad$ Pitchwise coordinate direction

$z \quad$ Spanwise coordinate direction

$\delta \quad$ Boundary-layer thickness

$\mu \quad$ Viscosity coefficient

$\rho \quad$ Density

$\sigma \quad$ Strain tensor 
$\tau \quad$ Domain flow-through time

Subscripts

$i \quad$ Inflow

$e \quad$ Exit or Outflow

\section{INTRODUCTION}

The quest for high efficiency in modern gas turbine engines is driving compressor overall pressure ratios and turbine inlet temperatures to much higher levels than in past designs. These harsher operating environments, with highly turbulent flow originating in the upstream stages and the combustion chamber spewing hot combustion products and other airborne particulates into the turbine stages, cause significant degradation of the turbine airfoil surfaces. The roughened airfoil surfaces lead to increased overall losses since the roughness adversely impacts the flow separation and transition process. Airfoil surface quality is also increasingly becoming an issue with the gradual shift to advanced ceramic matrix composite (CMC) or additivelymanufactured blades where achieving smooth surface finishes may be difficult. The ability to accurately predict details of the flow separation and transition process over rough airfoils is thus critical in order to properly design turbine airfoils to mitigate losses due to surface degradation and/or poor surface quality.

As the Reynolds numbers are typically low, the boundary layer on the suction surface of each airfoil in an LPT cascade initially remains laminar due to the high acceleration in the fore region of the airfoil, and generally separates just after the suction peak due to the adverse pressure gradient. The separated boundary layer typically undergoes transition, and the separated flow in the aft region of the airfoil is classified as "attached" or "open" depending on whether the boundary layer reattaches to form a separation bubble or does not reattach. "Open" separation leads to significant modifications to the overall airfoil pressure distribution and is responsible for higher losses and lower exit flow angles, while "attached" separation bubbles modify the pressure distribution only locally compared to the designed pressure distribution when the flow does not separate. "Attached" bubble topologies are obviously preferable in order to avoid performance detriments. The operating Reynolds number, flow angle, inflow turbulence level, and airfoil surface roughness dictate the suction surface separation characteristics in a LPT [1-3, and the references cited therein].

Scale-resolving simulation techniques, such as direct numerical simulation (DNS) and large-eddy simulation (LES), are increasingly becoming affordable due to the rapid growth in computational capability. Such techniques have the potential to accurately capture and predict the complex flow phenomena in turbomachinery and potentially lead the way to substantially improved designs. Several incompressible and compressible DNS studies (see, for example, [4-9]) and LES studies (see, for example, [10-14]) for turbine configurations have been reported in the

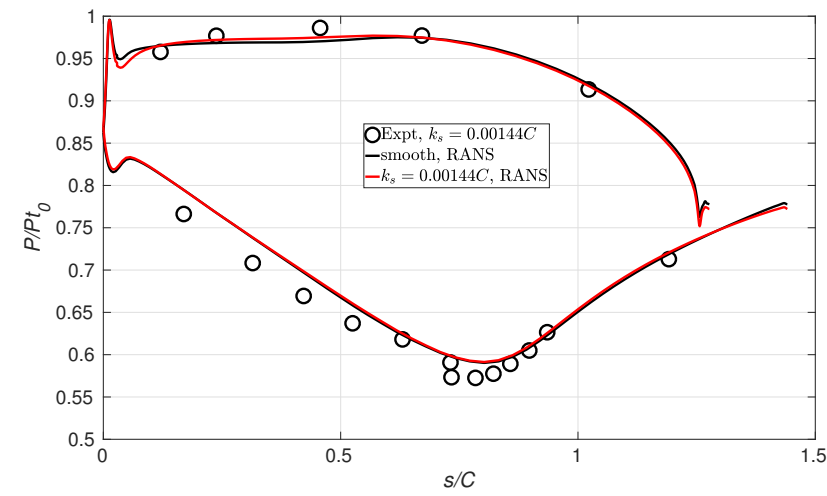

FIGURE 1: Surface pressure distribution for the smooth and $k_{s}=0.00144 C$ roughness airfoils from complementary RANS simulations conducted in the present study.

recent literature. These scale-resolving simulations have extensively studied effects such as the presence of inflow turbulence, incoming wakes, and rotor-stator interaction in turbomachinery assuming smooth airfoil surfaces.

More recently, Joo et al. $[15,16]$ have conducted LES studies of LPT and compressor cascade flows that incorporate the effect of wall roughness using discrete roughness elements or realistic roughness patterns. These studies have noted that simulations based on the Reynolds-averaged Navier-Stokes (RANS) equations fail to accurately capture the effects of roughness on the flow separation and transition characteristics. RANS simulations of the same LPT configuration that were conducted as part of the present study using the SST model [17] and the roughness model developed by Knopp et al. [18] showed that wall roughness had no influence on the blade loading (Fig. 1).

Discontinuous-Galerkin (DG) schemes for simulations of flows in complex geometries have recently been gaining attention due to their many attractive features, including their extensibility to arbitrarily high-order schemes on compact numerical stencils, applicability to different element types and mesh topologies, and computational efficiency. The use of DG methods in turbomachinery flow simulations is relatively new, and both RANS-based turbomachinery simulations [19-22] and DNS studies [23-28] using DG methods have been reported in the literature in recent years.

We have been developing an entropy-stable DG spectralelement method for the compressible Navier-Stokes equations, and in prior work have demonstrated its ability to predict laminar separation and transition in LPT airfoils and attached boundarylayer transition in a high-pressure turbine (HPT) airfoil cascade in the presence of inflow turbulence [26-28]. In the present work, we have extended the method to perform scale-resolving simulations of a roughened LPT airfoil cascade. The flow configuration 
is the same as that considered by Medic and Sharma [12] and Joo et al. [15] in their rough-wall LES studies, and for which experimental results have been reported by Sharma [29].

The paper is organized as follows. The numerical method is described briefly, followed by details of the flow configuration. Numerical results from the present simulations are then compared with experimental data and prior simulations, and the instantaneous behavior of the flow in the cascade is analyzed. Various aspects of the flow separation and transition process are also presented as a function of wall roughness, followed by some concluding remarks.

\section{NUMERICAL METHOD}

The compressible Navier-Stokes equations are solved using a space-time DG spectral-element method. In order to discretely satisfy the second law of thermodynamics (under exact integration), an entropy-variable formulation is used. The discretizations are formulated using piecewise polynomials of arbitrary order in both space and time directions to take advantage of tensor-product bases, and have been demonstrated up to $16^{\text {th }}$ order in both space and time. Integrals are approximated with a quadrature rule using twice the number of quadrature points as solution points in each spatial coordinate and temporal directions in order to minimize quadrature errors. The inviscid fluxes are computed using the entropy-stable approach of Ismail and Roe [30], while the viscous fluxes are computed using an interior penalty method where the penalty parameter is consistent with the second method of Bassi and Rebay [31]. The nonlinear system for each space-time slab is solved using a preconditioned Jacobian-free approximate Newton-Krylov solver. Further details regarding the space-time DG method can be found in [32-35]. Note also that no subgrid-scale or wall models are used in the present simulations.

In order to minimize spurious reflections from the inflow and outflow computational boundaries we have implemented the perfectly matched layer (PML) technique [36] in the numerical method. We have demonstrated in prior work $[27,37]$ that the PML technique for boundary specification leads to superior results for a variety of flow configurations including low-pressure turbine cascades.

Wall roughness is typically modeled in numerical simulations using either discrete geometric roughness elements (e.g., blocks, cylinders, hemispheres, etc.), or by incorporating actual roughness patterns obtained using surface imaging techniques. The latter approach is chosen in this work since it is more representative of actual operating conditions. Since we are dealing with a high-order flow solver that in turn requires high-order surface geometry information, the realistic surface roughness patterns are projected onto higher-order polynomials at the boundaries. We then use a high-order continuous-Galerkin approach to perturb the higher-order volume nodes using a linear-elasticity

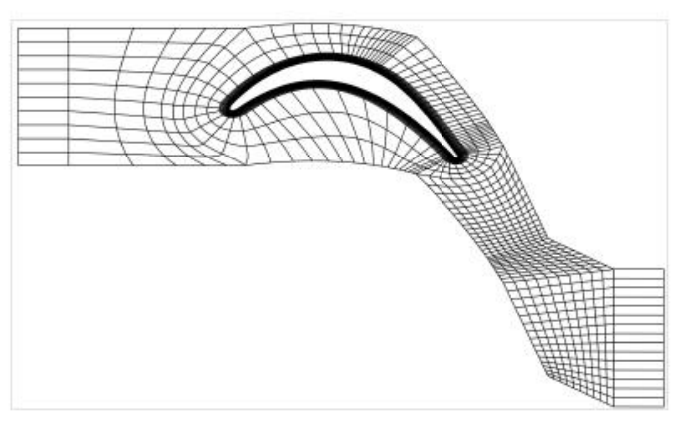

FIGURE 2: Computational mesh used in the smooth airfoil simulations.

analogy [38].

\section{PROBLEM SETUP}

The Pack109, Pack110, and Pack111 series of LPT airfoils have been studied over a wide range of Reynolds numbers in both experiments and large eddy simulations [12,15,29]. In this paper, we consider the Pack109 airfoil cascade geometry with an exit flow Reynolds number $R e=3.5 \times 10^{5}$. The inlet and exit Mach numbers are about 0.41 and 0.62 , respectively, and the inflow and exit flow angles with respect to the tangential direction are $46.8^{\circ}$ and $33.2^{\circ}$, respectively. [15].The experimentally-observed average centerline roughness (the arithmetical mean of the absolute value of the deviations from the mean line of the roughness profile), $R_{a}$, is $0.00024 C$. Following Joo et al. [15], we have used a factor of 6 to convert the $R_{a}$ value to an equivalent sand-grain roughness height, $k_{s}$. We note in this context that Bons [3] has suggested the use of $R_{a}$ to $k_{s}$ conversion factors ranging from 2 to 10 . In addition, we have also performed a simulation that assumes $k_{s}=R_{a}$.

The mid-span section of the airfoil passage is meshed with spatial elements of arbitrary order using a spectral isoparametric mapping technique in a multi-block configuration [26-28]. The spanwise extent of the computational domain is chosen as $0.2 C$, similar to the previous numerical studies by $[12,15]$, and is discretized using 16 elements. Periodic boundary conditions are used in both the pitchwise and spanwise directions, and an adiabatic wall boundary condition is used at the airfoil surface. Figure 2 shows the mesh used for the smooth airfoil case. The full blade passage is represented by about 24,000 elements. Using $8^{\text {th }}$ order elements in the spatial directions, the computational mesh results in about $12 \mathrm{M}$ degrees of freedom. This mesh has between $1-2$ elements in the suction side laminar boundary layer, and the effective wall-normal, streamwise, and spanwise spacings in wall units, $n^{+}, s^{+}$and $z^{+}$, of about 8,30 and 25 , re- 

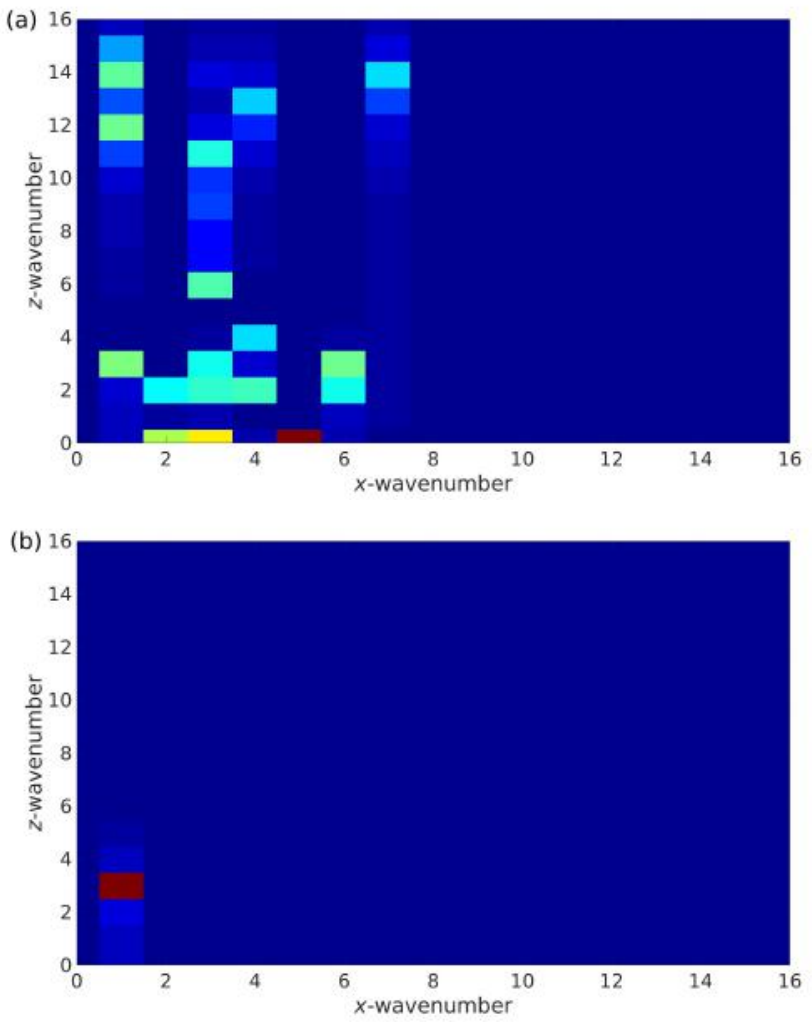

FIGURE 3: Perturbed modes included in the streamwise and spanwise directions to generate (a) Type A and B, and (b) Type $\mathrm{C}$ roughness in a $0.2 C \times 0.2 C$ patch. The color range from red to green to blue denotes decreasing energy for a given wavenumber.

spectively at $x \approx 0.96 C$ where the suction-side boundary layer is turbulent for the smooth airfoil case. This mesh is hereafter referred to as the 'coarse' mesh. We have also performed simulations with a refined mesh in the wall normal and streamwise directions in the vicinity of the suction peak, using a total of about 29600 elements, and 15M degrees of freedom for $8^{\text {th }}$ order elements in the spatial directions. This refined mesh has between $3-4$ elements in the suction side laminar boundary layer, and the effective $n^{+}, s^{+}$and $z^{+}$values are about 1,30 and 25 , respectively at the $x \approx 0.96 C$ streamwise location. This mesh is referred to as the 'fine' mesh.

The axial extent of the computational domain is chosen as $3 C$ in the present simulations. The domain flow-through time, $\tau$, is defined as the time taken by a fluid particle to travel from the inflow to the outflow boundary of the computational domain. The numerical simulations are performed with $4^{\text {th }}$ order implicit time stepping using a time-step of $2.5 \times 10^{-4} \tau$.

We have studied three different wall roughness types in an attempt to capture the full spectrum of wall roughness and un-

\begin{tabular}{c|c|c|c}
\hline \multirow{2}{*}{$\begin{array}{c}\text { Roughness } \\
\text { type }\end{array}$} & \multirow{2}{*}{$\begin{array}{c}\text { Maximum normal } \\
\text { displacement }(C)\end{array}$} & \multicolumn{2}{|c}{$k_{s}$ normalized by } \\
\cline { 3 - 4 } & 0 & 0 & $\delta_{x_{\text {suction }}=0.3 C}^{\text {smoot }}$ \\
\hline Smooth & 0.006 & 0.00144 & 0 \\
A & 0.001 & 0.00024 & 0.5 \\
B & 0.003 & 0.00144 & 0.08 \\
C & &
\end{tabular}

TABLE 1: Characteristics of different wall roughness patterns.

derstand its effects on the flow field. The meshes for the rough wall cases are generated from the smooth airfoil mesh using the linear-elasticity technique by perturbing it to generate the desired wall roughness. Since the actual scanned wall-roughness profiles are not available, the desired wall roughness pattern is generated using a patch of spatial extent of $0.2 C \times 0.2 C$ that includes various spectral modes of surface fluctuations. Figure 3 shows the perturbed wavenumbers to generate different roughness patterns. This patch is then applied repeatedly on the suction and pressure surfaces of the airfoil as a normal displacement to obtain a rough wall airfoil. As we are using 16 elements in the spanwise direction, we restrict the highest spanwise frequency to 14 , while in the streamwise direction, we restrict the highest frequency to 7 (Fig. 3-a). The resulting wall perturbations are then scaled to match the root mean square of the perturbations to $k_{s}=0.00144 C$ and $k_{s}=0.00024 C$. The wall-roughness pattern with $k_{s}=0.00144 C$ is referred to as Type A (Fig. 4-a), while the pattern with $k_{s}=0.00024 C$ is referred to as Type B (Fig. 4-b). We recognize that in practice resolving all the surface roughness elements may become computationally prohibitive and one may have to resort to resolving only certain portions or aspects of the roughness pattern and either modeling or ignoring the rest. To study this effect, we have also generated a third roughness pattern, Type C (Fig. 4-c), by considering the smallest streamwise and spanwise frequencies (Fig. 3-b), and then scaling to match the root mean square of the perturbations to $k_{s}=0.00144 C$. The normal displacements are smoothly zeroed out in the vicinity of the trailing edge where the radius is small to ensure that the suction and pressure surfaces do not collapse onto or cross each other. Table 1 provides more details regarding the characteristics of the wall roughness patterns used in this study. The generated "rough" airfoil surfaces are then used as boundary conditions in the linear-elasticity technique to perturb the high-order volume mesh. Figure 5 shows the resulting perturbed high-order volume mesh for the Type A wall roughness case. For roughness Type A and for the fine mesh on the suction side, the effective $n^{+}$and $s^{+}$ values vary from 1.5 and 100 at the fore section to 0.5 and 10 , respectively, near the trailing edge; for the coarse mesh, these values vary from 10 and 250 to 2 and 25 , respectively. 

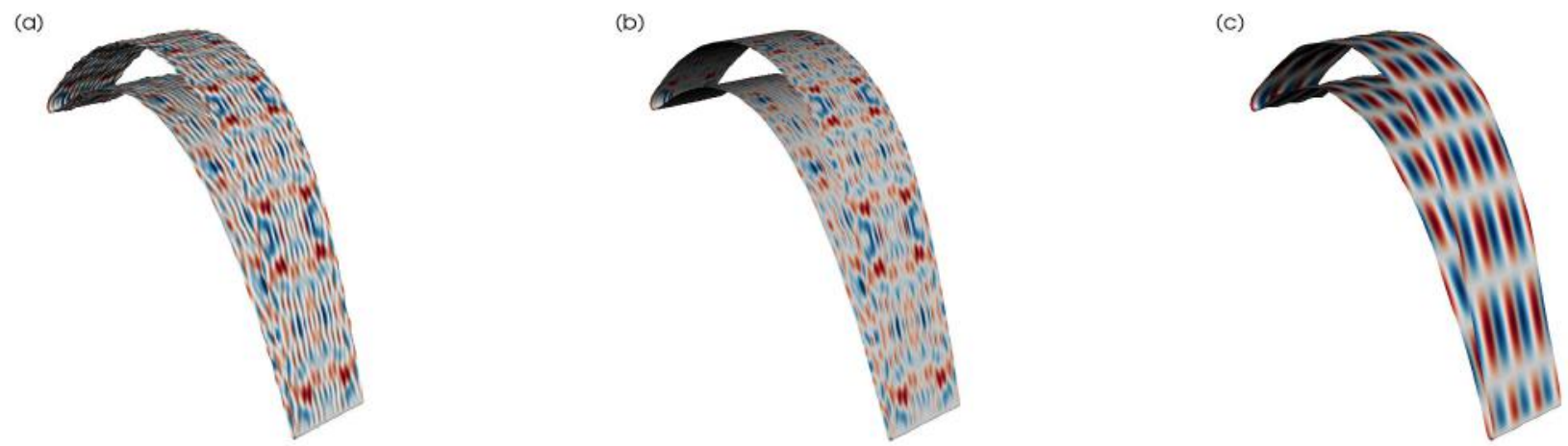

FIGURE 4: Wall roughness for (a) Type A, (b) Type B, AND (c) Type C. Red, white and blue regions denote positive, zero and negative normal displacements respectively.

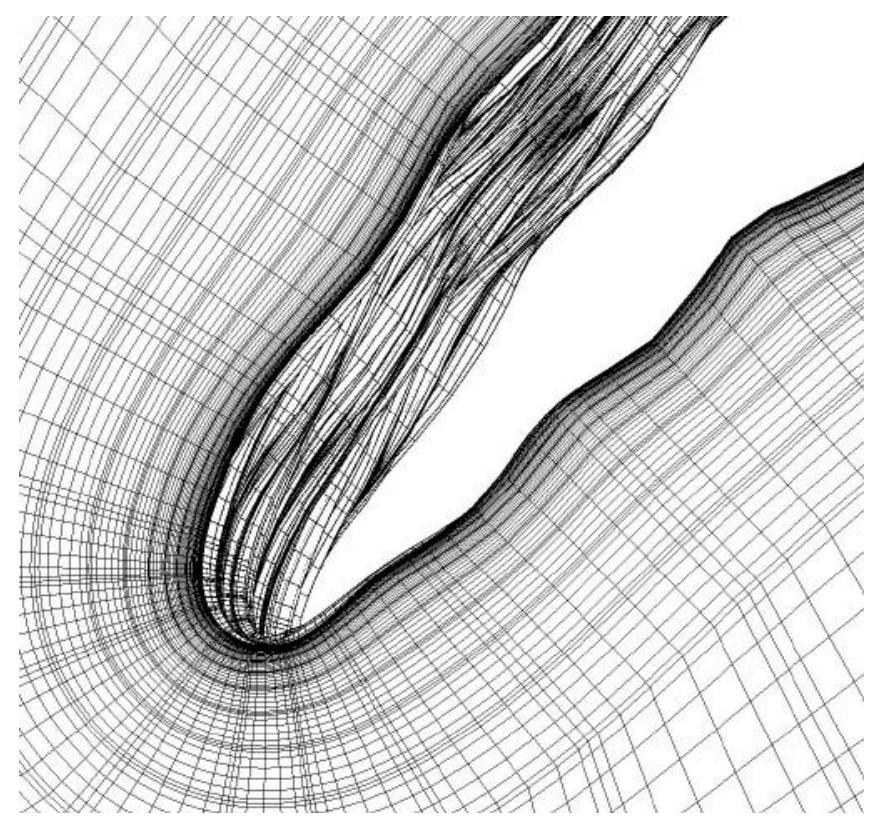

FIGURE 5: Computational mesh in the vicinity of the leading edge for Type A roughness. The leading edge region is magnified for visualization clarity purpose.

In addition to the scale-resolving simulations, we have also performed RANS simulations with the NASA OVERFLOW [39] solver in conjunction with the SST model [17] and the wall roughness model of Knopp et al. [18] using a structured oversetgrid approach to discretize the midspan section of the blade passage.

\section{NUMERICAL RESULTS}

Results from the scale-resolving and RANS simulations are presented in this section. For the scale-resolving simulations, flow stationarity is monitored using the temporal evolution of the airfoil surface pressure and skin friction. Mean flow statistics are then computed by averaging both in span and time over an additional $3 \tau$ for the coarse mesh, and $\tau$ for the fine mesh after the flow stationarity (temporal variation is less that $2 \%$ ) is achieved. The PML technique at the inflow and outflow boundaries was used for the fine mesh simulations only. Fine mesh simulations were performed for the smooth and roughness Type A airfoils, while coarse mesh simulations were performed for the smooth airfoil and for all three roughness types, A, B, and C.

\section{Grid Convergence}

Both the coarse and fine meshes result in similar mean surface pressure and skin friction distributions for the smooth and Type A roughness airfoils (Fig. 6). The suction-side separation bubble transitions and reattaches marginally further downstream for the fine mesh simulation compared to the coarse mesh simulation for the smooth airfoil. As the flow around the individual roughness elements is more resolved in the fine mesh, some small changes in the suction-side mean skin friction are noted. Unless otherwise noted, the results below for the smooth and roughness Type A airfoils are from the fine mesh simulations.

\section{Instantaneous Flow-Field Visualizations}

The instantaneous flow-fields in the LPT airfoil cascade are first examined using plots of the instantaneous Mach number on the $z=0$ plane (Fig. 7), and of the instantaneous skin friction coefficient on the airfoil surfaces (Figs. 8, 9).

On the suction surface of the airfoil, the flow rapidly accel- 

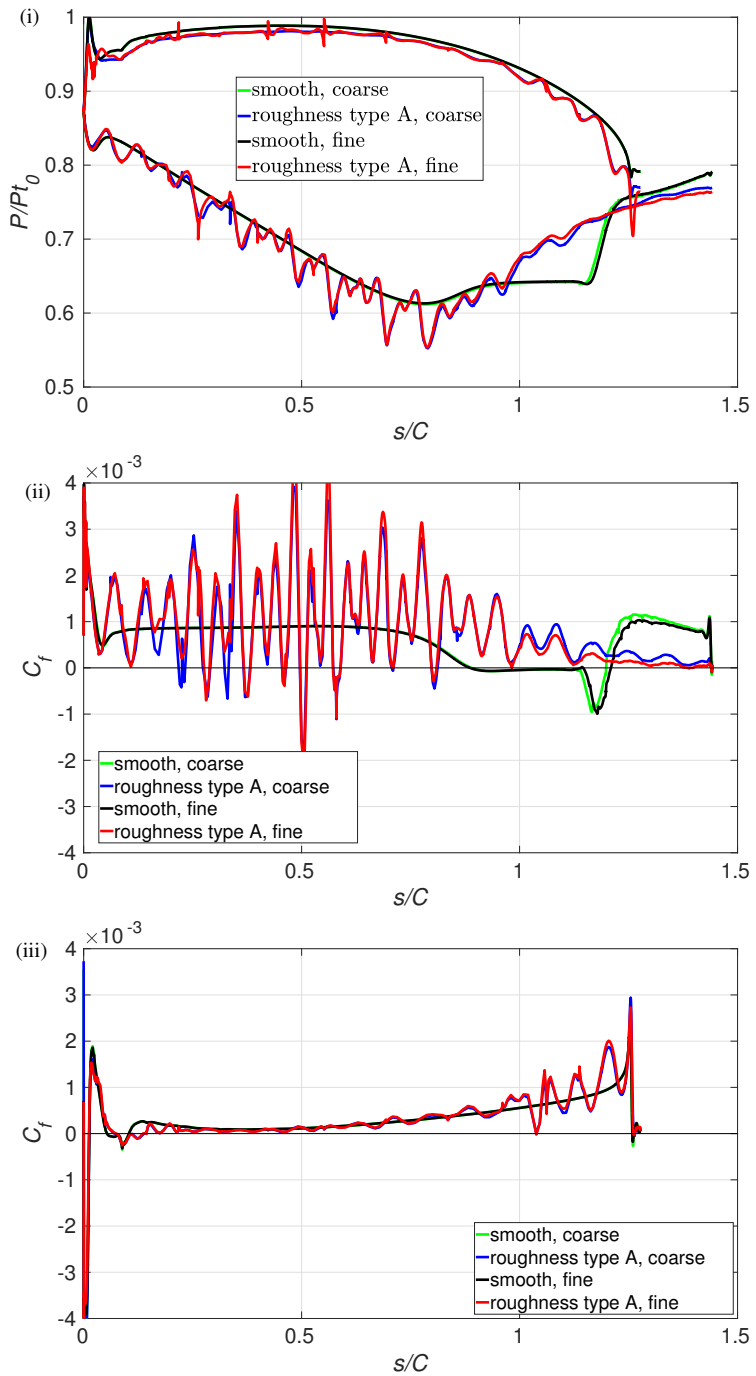

FIGURE 6: Comparison of (i) surface pressure; and skin friction on the (ii) suction, and (iii) pressure sides between coarse and fine mesh for different wall roughness.

erates from the leading edge to the suction peak and then decelerates downstream, while on the pressure surface the flow first decelerates in the fore region and then accelerates in the aft region of the airfoil. For the smooth airfoil, laminar flow separation occurs after the suction peak, the separated shear layer then transitions to turbulence, and the turbulent flow reattaches to the airfoil in the aft region. On the pressure surface, low intensity turbulence activity is also noticed (Fig. 7-a).

For the roughened airfoils, the flow transition characteristics vary widely depending on the wall roughness. With Type A wall roughness, the flow remains attached on the suction surface and the boundary layer can be seen to be turbulent even in the fore region of the airfoil. The turbulent suction surface boundary layer is much thicker, and much stronger turbulent activity is observed on the pressure surface when compared to the smooth airfoil case. The thicker turbulent boundary layers result in a thicker wake than the smooth airfoil case (Fig. 7-b). With Type $\mathrm{B}$ wall roughness where smaller roughness elements are present compared to Type A, and with Type $\mathrm{C}$ wall roughness where only large scale roughness elements are present (Table 1), the suction surface boundary layer remains laminar and attached until the suction peak. A much weaker laminar boundary-layer separation occurs on the suction surface compared to the smooth airfoil case. The separated laminar boundary then transitions and reattaches in the aft region of the airfoil. Similar to the smooth airfoil case, low intensity turbulence is observed on the pressure surface boundary layer. The turbulence intensity on the pressure surface is smallest for the Type B roughness case. The boundary-layer thickness and hence the width of the wake for both Type B and C roughness is similar to the smooth airfoil case, and thinner compared to the Type A case (Fig. 7-c, d).

The effect of the different wall roughness types on the separation characteristics of the suction surface boundary layers can also be visualized using the instantaneous skin friction coefficient, $C_{f}$ (Fig. 8). For the smooth airfoil, an "attached" separation bubble topology is observed (Fig. 8-a), consistent with the previous discussion (Fig. 7-a). The roughness pattern of the airfoil can be seen imprinted on the skin friction contours as regions with high and low $C_{f}$ values before and after the crest of the roughness elements, respectively. For Type A wall roughness, these localized high and low $C_{f}$ regions correspond to the flow attachment and separation around the roughness elements. The spanwise bands of localized separation and reattachment are due to the wall-roughness pattern since the crests of the roughness elements are aligned in the spanwise direction (Fig. 4-a). These localized separation regions lead to a turbulent boundary layer over the suction surface. Unlike the smooth airfoil case, the resulting turbulent boundary layer does not separate on the suction surface (Fig. 8-b). The separation bubble observed in the smooth airfoil case becomes weaker for the Type B and C roughness cases. The localized regions of separation and reattachment as observed with Type A roughness, are not present with Type B roughness since the roughness elements are smaller in size (Fig. 8-c); and the Type $\mathrm{C}$ roughness elements which also have much gentler slopes (Fig. 8-d) compared to Type A. Note also that for all roughness types, the roughness elements do not leave an imprint on the skin friction contours on the aft portion of the suction surface where the flow decelerates and becomes fully turbulent.

On the pressure surface, the boundary layer separates and reattaches near the leading edge for all airfoil roughness types (Figs. 9). Wall roughness modifies the shape of the leading edge and causes the separation bubble to become more three-dimensional (Figs. 9-b,c,d) compared to the smooth air- 

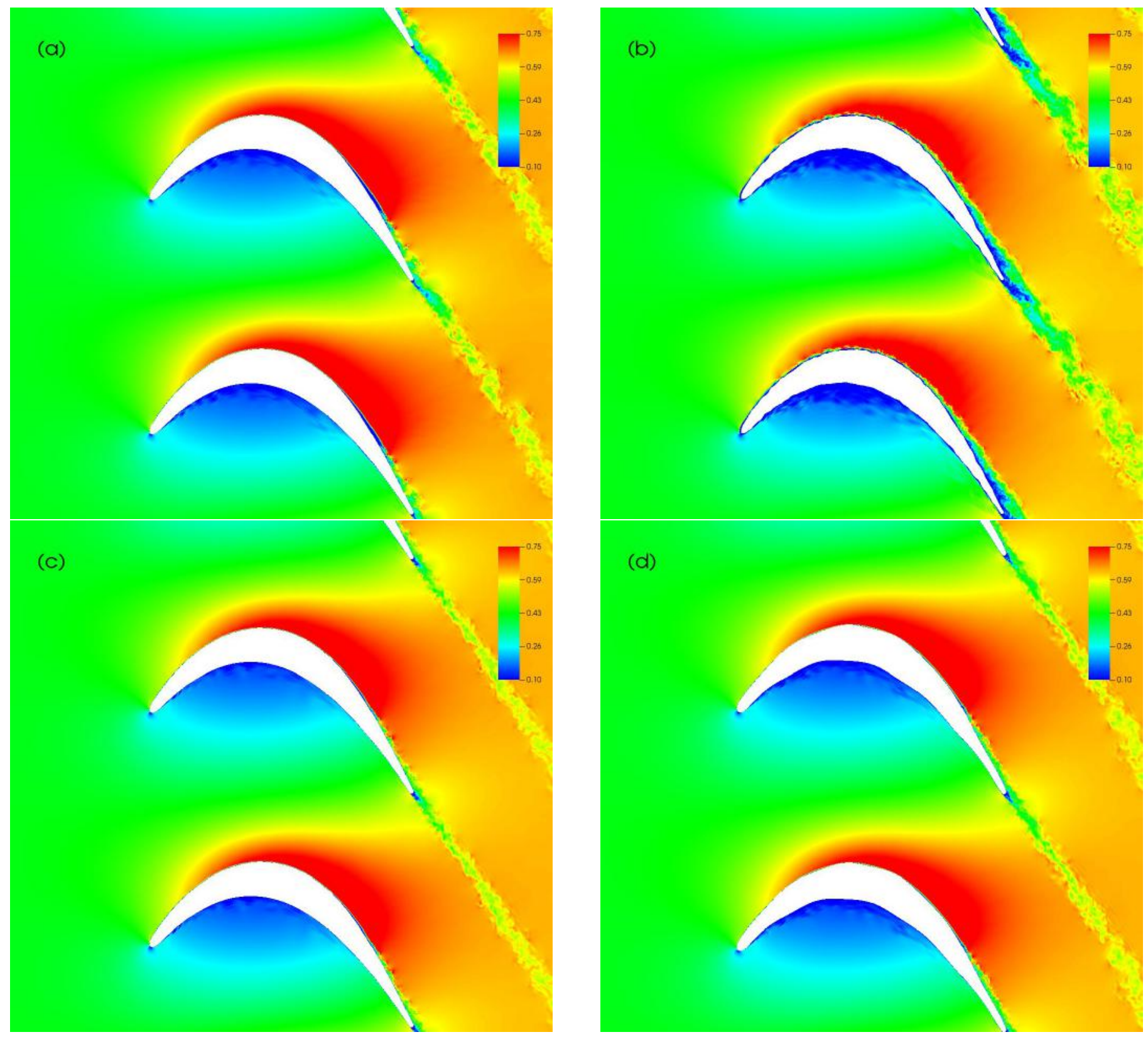

FIGURE 7: Instantaneous Mach number contours at the $z=0$ plane for (a) smooth airfoil, (b) Type A roughness, (c) Type B roughness, and (d) Type C roughness.

foil (Figs. 9-a). Turbulent structures of much higher intensity are noted on the pressure surface for Type A roughness (Fig. 9-b). For the smooth airfoil (Fig. 9-a), and for both Type B (Fig. 9-c) and Type $\mathrm{C}$ roughness (Fig. 9-d), long streaky streamwise structures are observed. Note that the turbulence on the pressure surface of the airfoil is not well resolved since we have used much larger elements here compared to the suction surface (Fig. 2).

\section{Airfoil Surface Pressure Distributions}

We next examine the mean surface pressure distributions on the airfoil obtained by averaging the instantaneous pressures in both the spanwise and temporal directions (Fig. 10-i) from the scale-resolving simulations. As seen in Figs. 7, 8, 9, the different wall roughness types influence the mean surface pressure distribution by modifying the boundary-layer separation and tran- 


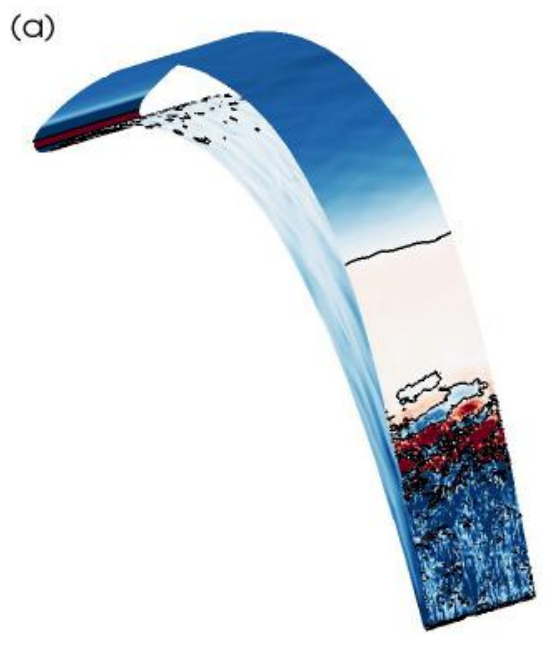

(c)

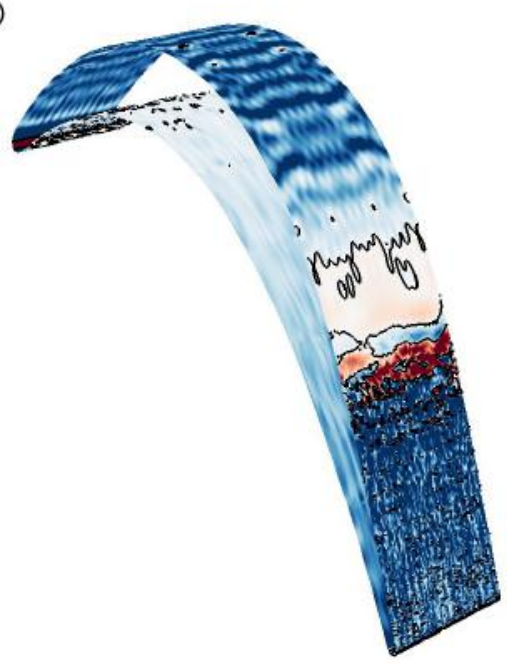

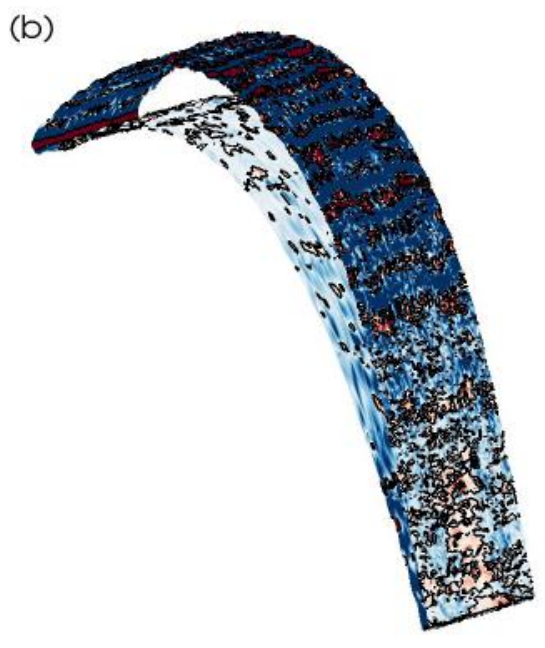

(d)

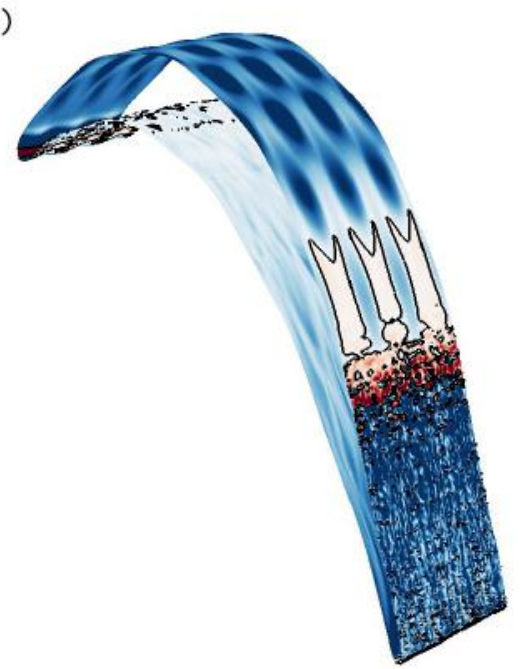

FIGURE 8: Instantaneous contours of $C_{f}$ for (a) smooth airfoil, (b) Type A roughness, (c) Type B roughness, and (d) Type C roughness on the suction surface of the airfoil. Blue and red color signify positive and negative $C_{f}$, respectively. $C_{f}=0$ is marked by the black lines.

sition characteristics. For the smooth airfoil case, an "attached" separation bubble forms in the aft region of the suction surface (Fig. 8-a) and the mean surface pressure is seen to plateau after the suction peak. The suction surface pressure recovers as the turbulent boundary layer reattaches. Since the suction surface separation bubble size is smaller for the Type $\mathrm{B}$ and $\mathrm{C}$ rough- ness airfoils (Figs. 8-c,d), the surface pressure recovers upstream compared to the smooth airfoil case. With Type A roughness, the mean surface pressure exhibits an oscillatory nature on the suction surface. This is due to the spanwise alignment of the local separation and reattachment regions induced by the roughness elements (Fig. 8-b). The results of Joo et al. [15] for a simi- 
(a)

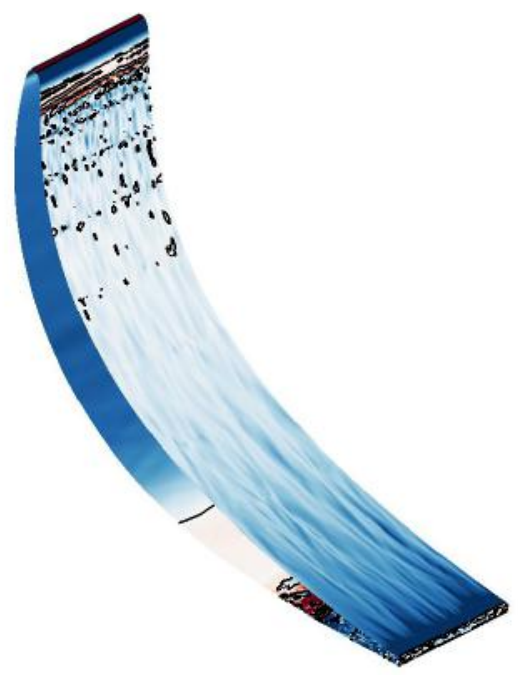

(c)

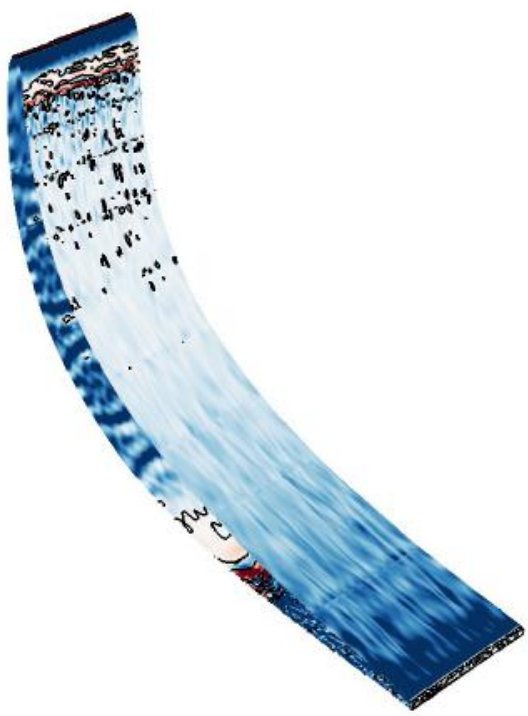

(b)

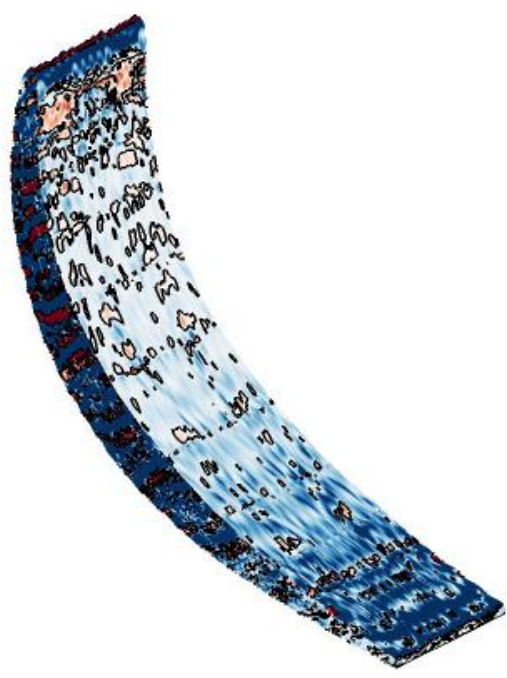

(d)

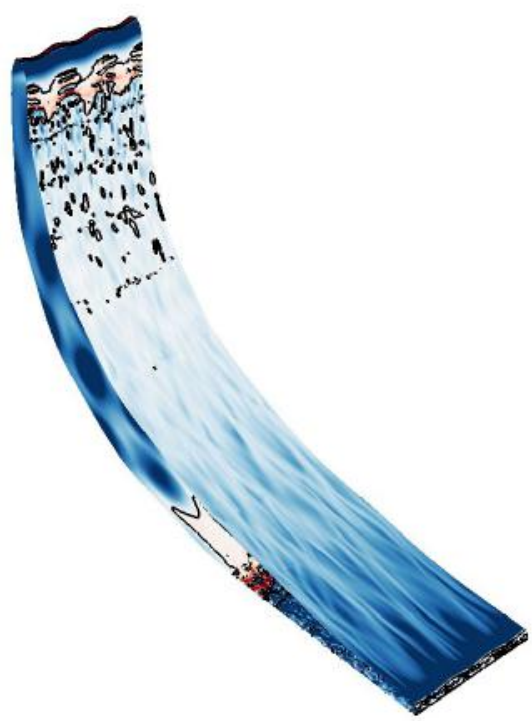

FIGURE 9: Instantaneous contours of $C_{f}$ for (a) smooth airfoil, (b) Type A roughness, (c) Type B roughness, and (d) Type C roughness on the pressure surface of the airfoil. Blue and red color signify positive and negative $C_{f}$, respectively. $C_{f}=0$ is marked by the black lines.

lar roughness are also plotted in the figure. Since they reported highly oscillatory surface pressure distribution at the suctionside fore section, their results are shown by the shaded region in Fig. 10-i. The surface pressure distribution from the present Type A roughness simulations agrees well with results of Joo et al. [15] with similar $k_{s}$. Since the boundary layer remains at- tached beyond the suction peak for the Type A roughness, the surface pressure exhibits a much smoother recovery compared to the other roughness and smooth airfoil cases. Note that roughness of any type does not seem to have any impact on the mean pressure distribution at the pressure surface. The mean surface pressures in the fore region of the suction surface are higher com- 

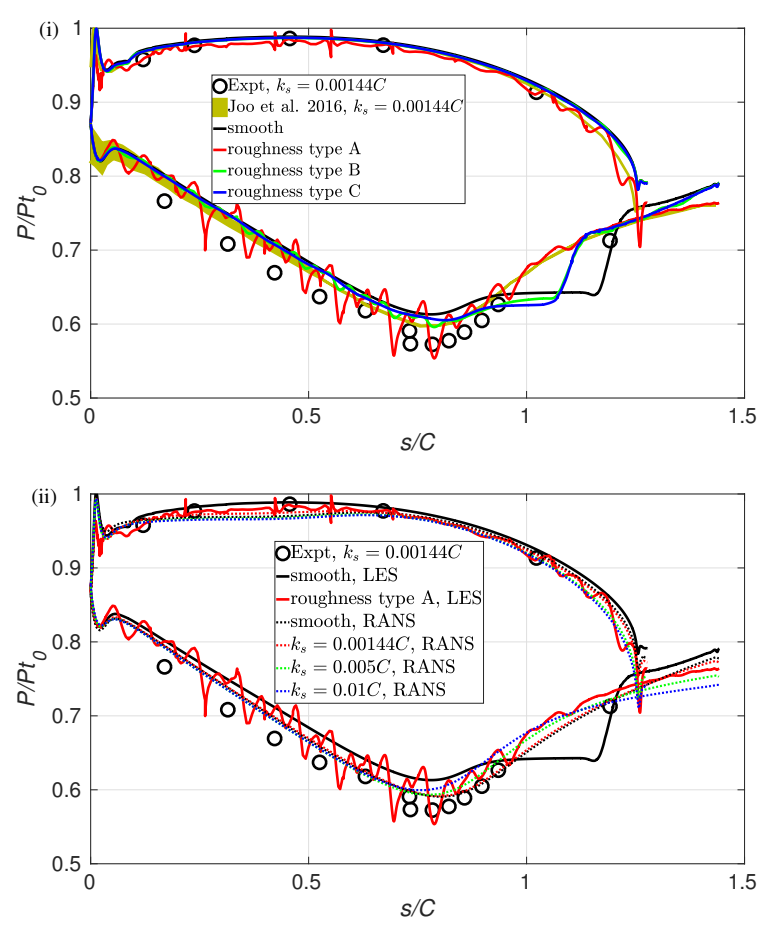

FIGURE 10: Mean airfoil surface pressure distributions for (i) different wall roughness in scale-resolving simulations, (ii) comparison between scale-resolving and RANS simulations.

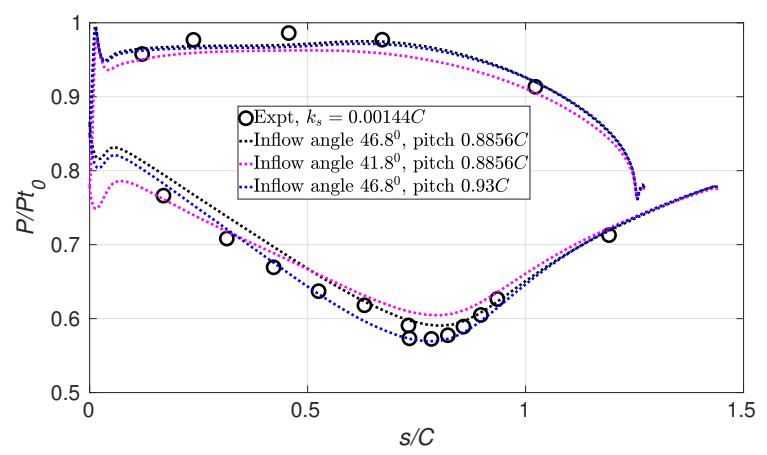

FIGURE 11: Airfoil surface pressure distribution from RANS simulations using different inflow angle and pitch.

pared to the experiments in both our simulations and those of Joo et al. [15] (Fig. 10-i).

The surface pressure distributions obtained from the scaleresolving simulations are compared to those from RANS simulations in Fig. 10-ii. Contrary to the scale-resolving simulations, the RANS simulations do not predict a suction-side separation bubble for the smooth airfoil, as it predicts fully turbulent bound- ary layer. When $k_{s}$ is increased to $0.00144 C$, the pressure distribution remains similar compared to the smooth RANS case. When $k_{s}$ is further increased, the pressure distribution starts to change at the suction-side aft section due to flow separation. The RANS simulations exhibit the same mismatch with the experiments as the scale-resolving simulations in the fore region of the airfoil suction surface.

In an attempt to understand the mismatch between the surface pressure distribution results from our simulations (both scale-resolving and RANS) and the experiment, we studied the effect of small changes in the inflow angle and the blade pitch on our RANS simulations. These results are shown in Fig. 11 and it is obvious that the pressure distribution is sensitive to these parameters. In particular, we note that a small $4 \%$ change in the blade pitch used in the simulations is enough to account for much of the discrepancy with the experiment. In addition, end-wall effects and other geometry variations could also contribute to the differences. However, rather than guess the values of the parameters used in the experiment, we have chosen to use the same values as reported in Joo et al. [15].

\section{Airfoil Skin Friction Distributions}

We next study the spanwise and time-averaged airfoil skin friction distributions (Fig. 12). In the case of the smooth airfoil, the suction-surface boundary layer separates at $s \approx 0.89 C$ and then reattaches at $s \approx 1.2 C$. As the separated shear layer undergoes transition, the mean skin friction distribution exhibits a plateau region followed by a region with much larger negative values around $s>1.1 C$ until the flow reattaches. Similar behavior of the skin friction distribution during flow transition in the separation bubble has also been reported in previous studies by [40-42, and references therein]. There is no separation bubble on the suction surface for the Type A roughness case; the roughness elements cause the mean skin friction to oscillate due to presence of the local regions of separation and reattachment around the roughness elements and sometimes even change sign. These oscillations in the mean skin friction are much less pronounced for Type $B$ and Type $C$ roughness than Type A roughness. Both Type $B$ and $C$ roughness cases exhibit smaller suction surface separation bubbles than the smooth airfoil, and reattachment is seen to occur at $s \approx 1.1 C$ in both cases. For the smooth airfoil and for both Type $\mathrm{B}$ and $\mathrm{C}$ roughness the skin friction decreases downstream after boundary-layer transition. For the rough airfoils, the mean skin friction oscillations on the suction surface are much stronger in the fore region where the flow is accelerating (Figs. 7) compared to the aft region where the flow is decelerating (Fig. 12-i).

Roughness Types B and C result similar suction-side separation-bubble characteristics, as they have the same large wavelength surface perturbation which results in similar unstable frequency for the separated shear layer. The small scale rough- 

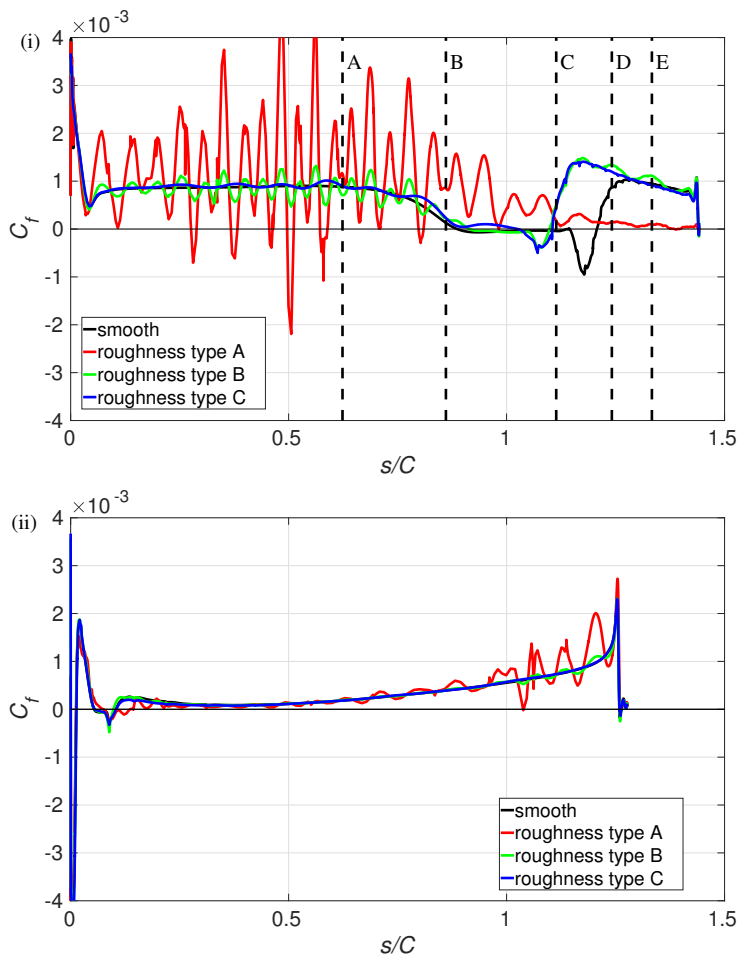

FIGURE 12: Mean skin friction distribution on the (i) suction, and (ii) pressure surface for different wall roughness. The black vertical dashed line in (a) denote the locations of the monitoring stations.

ness elements in the roughness Type A airfoil result the suctionside boundary layer to transition close to the leading edge, and no aft-section separation bubble. The smooth airfoil has the largest separation bubble due to absence of the surface roughness generated flow instabilities (Fig. 12-i).

As the suction and pressure surfaces of the airfoil are delineated by the location where the wall normal in the pitchwise direction changes sign, the leading edge stagnation point does not coincide with the starting location of the suction or pressure surface. Instead, the leading edge stagnation point occurs on the pressure surface (Fig. 10), causing the skin friction on the pressure surface for $s<0.01 C$ to be negative with attached flow (Figs. 9, 12-ii). A small separation bubble on the pressure surface near the leading edge exists for all the roughness types considered. This separation bubble extends from $0.05 C<s<0.1 C$ for the smooth airfoil and for both Type B and $\mathrm{C}$ roughness, and between $0.07 C<s<0.1 C$ for Type A roughness due to the modification of the leading edge geometry (Figs. 9). As in the case of the suction surface, for the rough airfoils oscillations in the mean skin friction are much stronger close to the trailing edge where the flow is accelerating (Figs. 7) compared to the leading edge regions where the flow is decelerating on the pressure surface (Fig. 12-ii) .

\section{Suction-Surface Boundary-Layer Characteristics}

We next study the turbulence characteristics of the suction surface boundary-layer by analyzing the Favre-averaged mean velocities and Reynolds stresses. The boundary-layer thickness, $\delta$, is computed as the distance from the wall where the mean tangential velocity reaches $0.99 U_{\infty}$.

For the smooth airfoil, the turbulence intensity attains nonzero values inside the boundary layer in the aft region of the suction surface as the flow transitions in the separated boundary layer (Fig. 13-a). The turbulent kinetic energy peaks during the transition process and decreases downstream. The boundary layer thickness remain constant $(0.003 C)$ for $0.1 C<x<0.6 C$ and then increases due to boundary-layer separation and transition. The boundary-layer thickness increases more rapidly as it becomes turbulent compared to the separated laminar boundary layer. Note that the value of $k_{s}$ is $\approx 0.5$ of the smooth airfoil boundary-layer thickness in the fore region of the suction surface for Type $\mathrm{A}$ and $\mathrm{C}$ roughness while it is $\approx 0.08$ of the boundarylayer thickness for the Type B roughness (Table. 1). The largest roughness elements are about two times, one-third, or the same size of the suction surface region boundary-layer thickness for the smooth airfoil, roughness Type $\mathrm{A}$ and $\mathrm{B}$, and roughness Type $\mathrm{C}$, respectively.

For Type A roughness, high turbulent intensity values are observed inside the boundary layer over the entire suction surface (Fig. 13-b). The local separation and transition regions induced by the roughness elements result in patches of high turbulence regions in the fore region of the airfoil. Stronger patches of high turbulent intensity occur around the suction peak $(0.4 C<x<0.65 C)$, beyond which the turbulent intensity decreases as the flow decelerates. In comparison to the smooth airfoil, the boundary-layer thickness is larger for the Type A roughness.

The Type B roughness case (Fig. 13-c) has much lower turbulent intensity in the fore region of the suction surface than the Type A case (Fig. 13-b) since the roughness elements are smaller in size than the former. For Type $\mathrm{C}$ roughness, the high turbulent intensity region in the fore region of the suction surface (Fig. 13d) is mostly due to laminar flow adjustment around the largescale spanwise inhomogeneity (Fig. 4-c). The boundary-layer thickness in the suction-surface fore region for Type B and C roughness is similar to the smooth airfoil. Since the suction surface separation bubble is smaller compared to the smooth airfoil for these roughened airfoils, the boundary-layer thickness in the aft region is also smaller compared to the smooth airfoil.

We have selected five monitoring stations to investigate the mechanism of boundary-layer transition on the suction surface of the airfoils in greater detail (Fig. 12-a, 13). For the smooth 

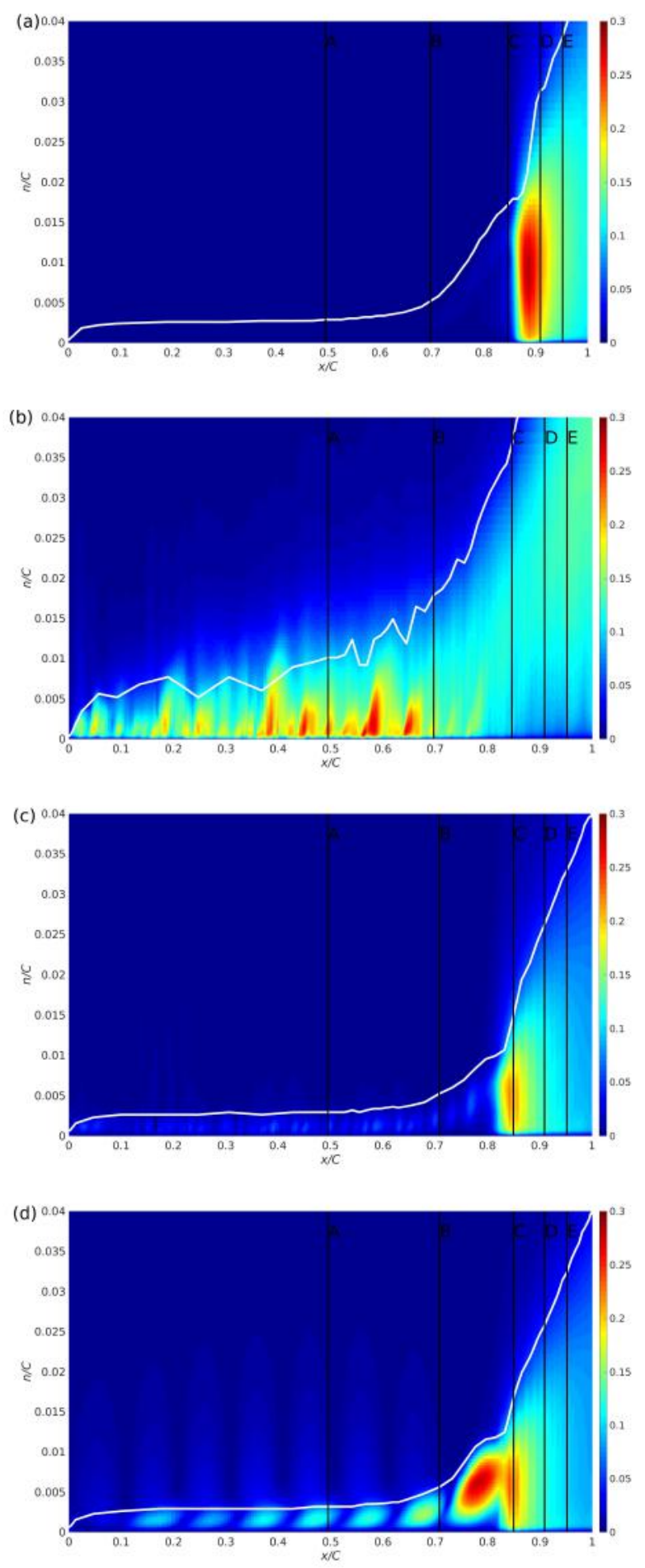

FIGURE 13: Turbulent intensity contours on the airfoil suction surface for (a) smooth airfoil, (b) Type A roughness, (c) Type B roughness, and (d) Type $\mathrm{C}$ roughness. The black vertical lines denote the locations of the monitoring stations as shown in Fig. 12a, ant the white line denotes the edge of the boundary layer. airfoil, the monitoring station $\mathrm{A}$ is located in the attached laminar boundary layer, station B is located just prior to laminar boundary-layer separation, station $\mathrm{C}$ is located at the initial stage of transition, station D is located at the final stage of transition, and station $\mathrm{E}$ is located in the turbulent region. For Type $\mathrm{A}$ roughness, all the monitoring stations are located in the turbulent region, and for Type $\mathrm{B}$ and $\mathrm{C}$ roughness, station $\mathrm{A}$ is located in the attached boundary layer, station $\mathrm{B}$ is located just prior to boundary-layer separation, station $\mathrm{C}$ is located at the flow reattachment, and stations $\mathrm{D}$ and $\mathrm{E}$ are located in the turbulent region. Note that the monitoring stations $\mathrm{A}$ and $\mathrm{B}$ are around the suction peak, while the rest of the stations are in the region where the flow is decelerating.

The mean tangential velocity profiles (Fig. 14-i), $\widetilde{U}_{s}$, are similar at stations $\mathrm{A}$ and $\mathrm{B}$, upstream of the suction peak, for the smooth and both Type $\mathrm{B}$ and $\mathrm{C}$ roughness airfoils, but the Type A roughness case exhibits a much thicker boundary layer compared to the other cases. The local separation and reattachment regions induced by the Type A roughness elements result in enhanced momentum transfer and hence a thicker boundary layer. The $\widetilde{U}_{s}$ values at the local edge of the boundary layer are lower at the downstream stations $\mathrm{C}-\mathrm{E}$, which are located in the flow decelerating region. Since the smooth airfoil has a larger suction-surface separation bubble compared to the Type B and $\mathrm{C}$ roughness cases (Fig. 12-i), the $\widetilde{U}_{s}$ profiles at station $\mathrm{C}$ indicate reversed flow for the smooth airfoil, and thinner boundary layers at the stations D and E for Type B and C wall roughness. Note also that the Type A roughness case shows much stronger flow deceleration compared to the other cases.

For the smooth airfoil, the turbulent kinetic energy is zero at the monitoring stations $\mathrm{A}$ and $\mathrm{B}$ since the boundary layer is laminar (Fig. 14-ii,iii,iv). At station C, the tangential component of turbulent kinetic energy peaks inside the separated shear layer, close to the boundary-layer edge. After flow reattachment, the tangential and spanwise components remain almost constant and their values are similar for most of the boundary layer at stations D and E. In the simulations of flows over a wall-mounted cubes or periodic hills by Hussein and Martinuzzi [43], Breuer et al. [44], Diosady and Murman [33], tangential component of the turbulent kinetic energy peaks at the shear layer originating from the crests of the cubes or hills. In present simulations, we observe that at station A the tangential component peaks at the same height for both Type A and B roughness even though the $k_{s}$ values are different for the two roughness types. The peak in the tangential component for the wall roughness type $\mathrm{C}$ at monitoring stations A and B is mostly due to spanwise inhomogeneity rather than temporal unsteadiness, as described earlier. The peak value of the tangential component decreases from station A to B for Type $\mathrm{A}$ and $\mathrm{B}$ roughness. At stations $\mathrm{C}-\mathrm{E}$ the tangential and spanwise components remain almost constant for most of the boundary layer for the rough airfoils. Also, for Type B and $\mathrm{C}$ roughness, the turbulent kinetic energy components at station 

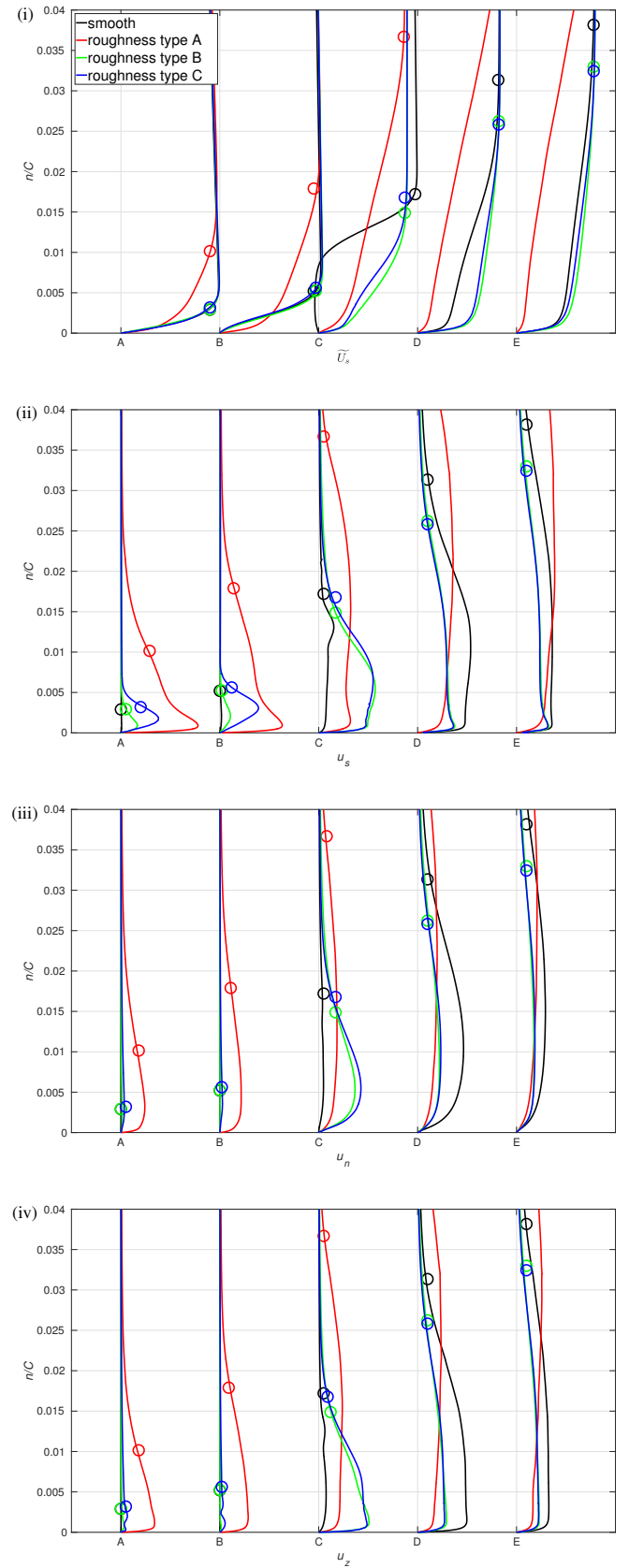

FIGURE 14: Wall normal profiles of (i) mean tangential velocity, and (ii) tangential, (iii) wall normal, and (iv) spanwise component of turbulent intensity. The black, red, green, and blue color lines denote the smooth airfoil and roughness Types A, B, and C, respectively. The open circles denote the edge of the boundary layer.
$\mathrm{C}$ is larger in comparison to either the smooth airfoil or Type $\mathrm{A}$ roughness case. As one would expect, the wall normal component increases more slowly with the wall normal distance compared to the tangential and spanwise components.

Turbulent Kinetic Energy Budget We next study the resolved turbulent kinetic energy budgets for both the smooth and rough airfoil cases to glean insights that can potentially help to inform the wall-roughness modeling efforts for RANS and/or LES. The turbulent kinetic energy budget for compressible flow that has attained stationarity can be written as:

$$
0=M C+P+D+P s+P t+T t+V t+M,
$$

where the right hand side terms in order are the mean convection, production, dissipation, pressure-strain, pressure transport, turbulent transport, viscous transport, and mass flux variation, respectively [45]. These terms are defined as:

$$
\begin{gathered}
M C=-\frac{\partial}{\partial x_{k}}\left(\tilde{u_{k}} \bar{\rho} \widetilde{u_{i}^{\prime \prime} u_{i}^{\prime \prime}}\right), \\
P=-\bar{\rho} \widetilde{u_{i}^{\prime \prime} u_{k}^{\prime \prime}} \frac{\partial \tilde{u}_{i}}{\partial x_{k}}, \\
D=-\overline{\sigma_{i k}^{\prime} \frac{\partial u_{i}^{\prime}}{\partial x_{k}}}, \overline{\frac{\partial u_{k}^{\prime}}{\partial x_{k}}} \\
P s=-\frac{\partial \overline{p^{\prime} u_{k}^{\prime}}}{\partial x_{k}}, \\
T t=-\frac{\partial \bar{\rho} u_{i}^{\prime \prime} u_{i}^{\prime \prime} u_{k}^{\prime \prime}}{\partial x_{k}}, \\
V t=\frac{\partial \overline{\sigma_{i k}^{\prime} u_{i}^{\prime}}}{\partial x_{k}}, \\
M=\overline{u_{i}^{\prime \prime}}\left(\frac{\partial \overline{\sigma_{i k}}}{\partial x_{k}}-\frac{\partial \bar{p}}{\partial x_{i}}\right), \\
\sigma_{i j}=\mu\left(\frac{\partial u_{i}}{\partial x_{j}}+\frac{\partial u_{j}}{\partial x_{i}}-\frac{2}{3} \frac{\partial u_{k}}{\partial x_{k}} \delta_{i j}\right),
\end{gathered}
$$

where the overbar and tilde represent the Reynolds-averaged and Favre-averaged values, respectively, and / and // represent the deviations from the Reynolds- and Favre-averages, respectively. For the present simulations, the mass flux variation term, $M$, is omitted from the figures since the compressibility effect $\left(\overline{u_{i}^{\prime \prime}} \approx 0\right)$ is negligible.

For the smooth airfoil, at station $\mathrm{C}$ (located in the early stages of transition) the turbulent production term is zero in much 

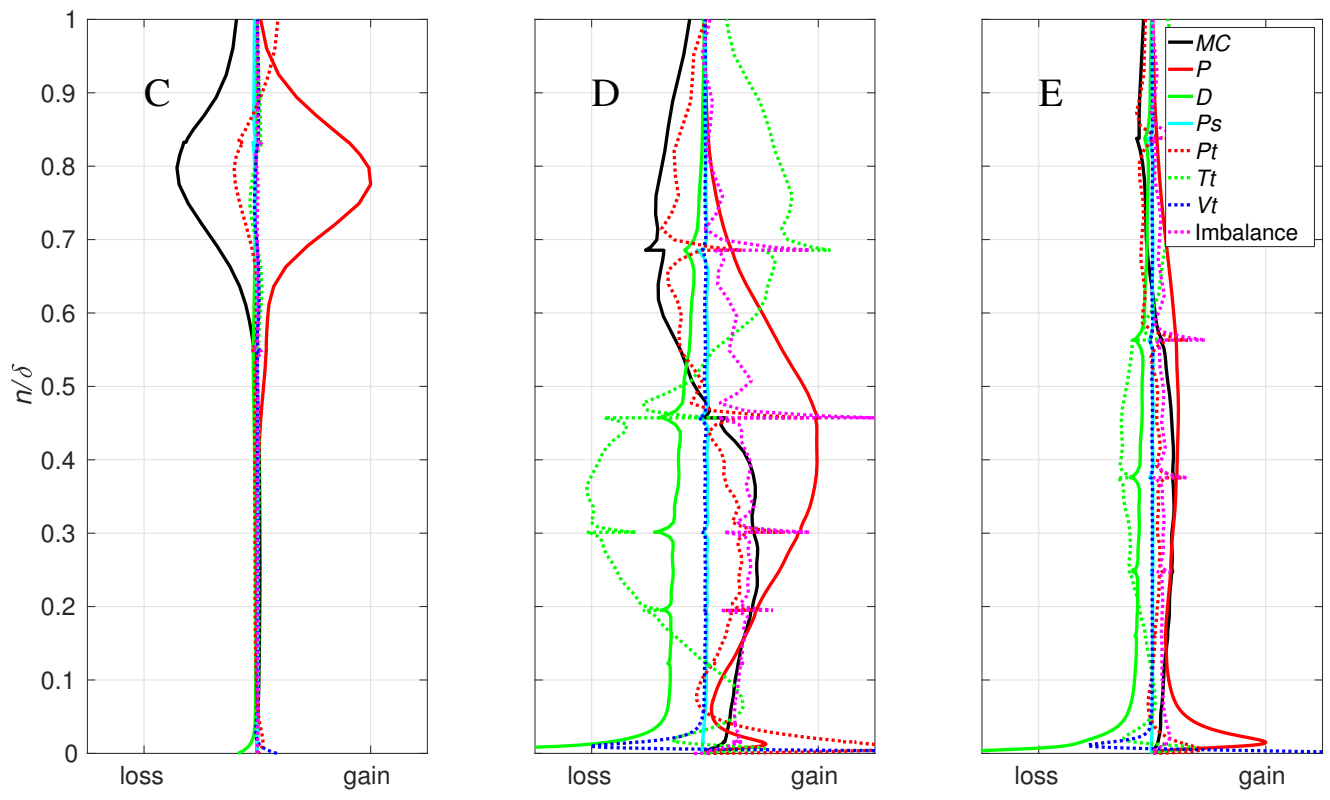

FIGURE 15: Wall normal profiles of the resolved turbulent kinetic energy budget terms at stations $\mathrm{C}, \mathrm{D}$, and $\mathrm{E}$ for the smooth airfoil. Budget terms are normalized by the peak production at each station.

of the boundary layer except at the separated shear layer. Turbulence production peaks inside the separated shear layer, and is balanced by the mean convection and pressure transport. At the edge of the boundary layer, the pressure transport is balanced by the mean convection. The downstream stations $\mathrm{D}$ and $\mathrm{E}$ are located after the flow has transitioned and reattached (see Fig. 12a). At station $D$ the turbulent production peaks close to the airfoil and in the mid-region of the boundary layer. Mean convection and pressure transport act as source terms in the near-wall regions of the boundary layer, but act as sink terms in the top region away from the airfoil. Turbulent transport is observed to behave in a manner that is the opposite of the mean convection. Dissipation is highest close to the airfoil, and gradually decreases to zero away from the airfoil. At station E all the energy budget terms are negligible over the boundary layer except close to the wall. Turbulent production peaks close to the airfoil and is balanced by dissipation (Fig. 15).

The turbulent energy budget behavior is markedly different with Type A roughness in comparison to the smooth airfoil. The turbulent production, pressure transport and mean convection are the dominant source terms near the wall, and they are balanced by the dissipation and turbulent transport at monitoring stations $\mathrm{A}$ and $\mathrm{B}$. The contribution from the mean convection are smaller at stations $\mathrm{C}-\mathrm{E}$ compared to stations $\mathrm{A}$ and $\mathrm{B}$. At stations $\mathrm{C}-\mathrm{E}$ turbulent production is the dominant source term, and is balanced by the dissipation, mean convection, turbulent transport, and pressure transport across much of the boundary layer (Fig. 16).

With Type B and C roughness, the suction surface separation bubbles are much smaller and the flow transitions earlier compared to the smooth airfoil (Fig. 12-i). The monitoring station C is located in the turbulent reattachment region, while the monitoring stations D and E are located further downstream. Consequently, for both these roughness cases the turbulent kinetic energy budget at monitoring station $\mathrm{C}$ is similar to the monitoring station D of the smooth airfoil, and at monitoring stations D and $\mathrm{E}$ is similar to the monitoring station $\mathrm{E}$ of the smooth airfoil. The turbulent kinetic energy budget profiles for these cases are hence not included in the paper for the sake of brevity.

The turbulence budget (Figs. 15 and 16) are not fully balanced since the present simulations do not resolve all the turbulent scales. Even very close to the wall, where $n^{+} \approx 1$, the turbulent budget terms are not balanced, due to the lack of streamwise and spanwise resolution. The turbulence budget term plots, Figs. 15 and 16, exhibit jumps at the element boundaries, particularly close to the boundary-layer edge where the element size is large. This is due to the inherent nature of the DG approach used and underscores the need for an adjoint-driven unstructured mesh adaptation technique. The development of chaotic adjoints for turbulent flows [46] and high-order unstructured hex-dominant 

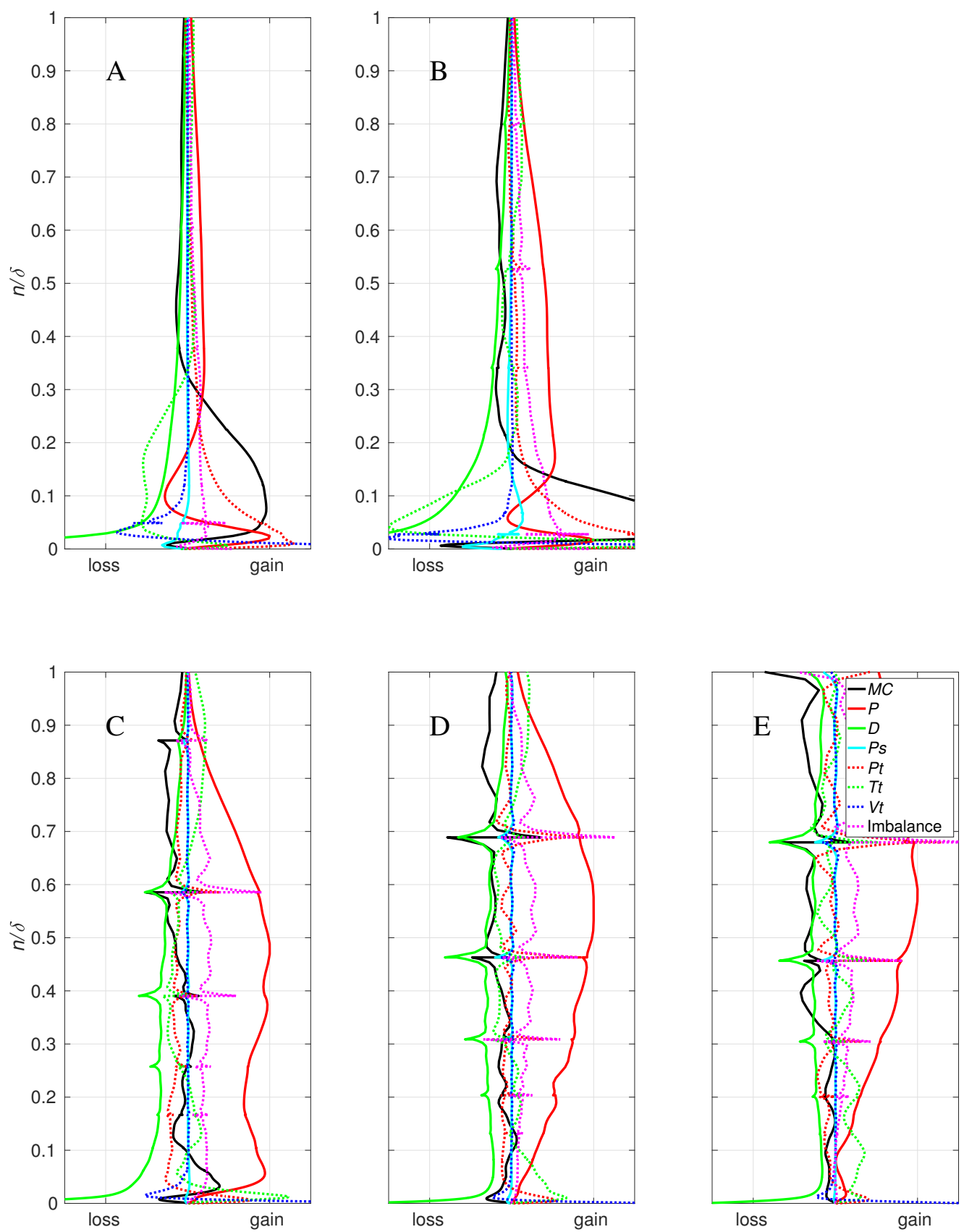

FIGURE 16: Wall normal profiles of the resolved turbulent kinetic energy budget terms at stations A - E for Type A roughness. Budget terms are normalized by the peak production at each station.

mesh generation tools [47] are the focus of current research.

\section{CONCLUDING REMARKS}

We have used an entropy-stable spectral-element formulation to perform scale-resolving simulations of flow in an LPT 
cascade with rough airfoils. The results show that the full spectrum of wall-roughness significantly influences the flow separation and transition characteristics. For the Pack109 geometry chosen here, smooth airfoil simulations show a laminar separation bubble on the suction surface that then transitions and reattaches in the aft region. The Type B roughness (wall roughness with $k_{s}=0.00024 C$ ), and the Type C roughness (large scale only wall roughness with $k_{s}=0.0014 C$ ) modify the suction surface separation bubble. Both of them have smaller separation bubbles resulting in thinner suction surface boundary layer at the aft section compared to the smooth airfoil. For the Type A roughness $\left(k_{s}=0.0014 C\right)$ the suction surface boundary layer remains attached, and it transitions at the fore section due to localized separation and reattachment regions from the large roughness elements. Hence, the suction surface boundary layer and wake are thicker for this airfoil compared to the smooth and Type B and C roughness airfoils.

The smooth and Type A roughness cases predict the the separated and attached suction-side boundary layer respectively, consistent with the experiment and previous simulations by Joo et al. [15]. Although present simulations overpredict surface pressure at the suction-side fore section, consistent with the previous simulations by Joo et al. [15]. RANS simulations also result in similar mismatch, and the mismatch decreases as the inflow angle decreases, or blade pitch increases. End-wall effects, uncertainty in geometry definition, flow conditions etc. may also effect the airfoil loading beside inflow angle and pitch. RANS simulations fail to capture the suction-side boundary-layer characteristic as a function of surface roughness. RANS simulation of the smooth airfoil does not predict any suction-side separation bubble. The surface pressure distribution by the RANS simulations is similar for the clean and $k_{s}=0.0014 C$ roughness case. he The suction-side aft-section pressure distribution in the RANS simulations with larger $k_{S}$ is modified due to occurrence of a separation bubble.

We have also attempted to contrast the simulations with different wall roughness in some detail by analyzing the transitional and turbulence nature of the flow fields. The turbulent intensity profiles and the turbulent kinetic energy budgets reveal that the wall roughness characteristics influence the flow transition behavior. The turbulent kinetic energy budget indicates that the turbulence is not fully resolved for these simulations.

The results presented here demonstrate that the capability of the spectral-element formulation to capture the effect of wall roughness in a LPT cascade. The present simulations clearly demonstrate the influence of both the "resolved" roughness element height and length scales on the boundary-layer separation and transitional characteristics of the flow. In practice, simulations that fully resolve the wall-roughness elements can become prohibitively expensive from a computational standpoint, and the wall roughness will may to only be partially resolved while the remainder is modeled or ignored. The Type A and
$\mathrm{C}$ roughness cases elucidate the influence of the resolved roughness on the boundary layer transition and loss characteristics, and can be used as a benchmark of the unresolved surface roughness modeling. Although no wall models are used in present simulations, these computations represent a first step toward developing a more general capability that incorporates roughness effects for "wall-modeled" and/or "wall-resolved" LES that can help reduce the computational expense of such scale-resolving simulations.

\section{ACKNOWLEDGMENT}

Funding for Anirban Garai was provided by the Advanced Air Transport Technology Project in the NASA Advanced Air Vehicles Program through Science and Technology Corporation. Additional funding for this work was provided by the Transformational Tools and Technologies Project in the NASA Transformative Aeronautics Concepts Program. Computing resources were provided by the NASA Advanced Supercomputing facility at NASA Ames Research Center. The authors wish to acknowledge Dr. Om Sharma of United Technologies Research Center for providing the airfoil geometry and experimental conditions and for many helpful discussions.

\section{REFERENCES}

[1] Mayle, R. E., 1991. "The role of laminar-turbulent transition in gas turbine engines". ASME Journal of Turbomachinery, 113, pp. 509-537.

[2] Michalek, J., Monaldi, M., and Arts, T., 2012. "Aerodynamic performance of a very high lift low pressure turbine airfoil (T106C) at low Reynolds and high Mach number with effect of free stream turbulence intensity". ASME Journal of Turbomachinery, 134, p. 061009.

[3] Bons, J. P., 2010. "A review of surface roughness effects in gas turbines". ASME Journal of Turbomachinery, 132, p. 021004.

[4] Wu, X., and Durbin, P. A., 2001. "Evidence of longitudinal vortices evolved from distorted wakes in a turbine passage". Journal of Fluid Mechanics, 446, pp. 199-228.

[5] Wissink, J. G., and Rodi, W., 2006. "Direct numerical simulation of flow and heat transfer in a turbine cascade with incoming wakes". Journal of Fluid Mechanics, 569, pp. 209-247.

[6] Rai, M. M., 2006. "A direct numerical simulation of transitional and turbulent flow on a turbine airfoil". AIAA Paper 2006-4460.

[7] Sandberg, R. D., Pichler, R., Chen, L., Johnstone, R., and Michelassi, V., 2015. "Compressible direct numerical simulation of low-pressure turbines: part I - methodology". Journal of Turbomachinery, 137(5), p. 051011.

[8] Michelassi, V., Chen, L., Pichler, R., and Sandberg, R. D., 
2015. "Compressible direct numerical simulation of lowpressure turbines: part II - effect of inflow disturbances". Journal of Turbomachinery, 137(7), p. 071005.

[9] Michelassi, V., Chen, L., Pichler, R., Sandberg, R. D., and Bhaskaran, R., 2016. "High-fidelity simulations of lowpressure turbines: effect of flow coefficient and reduced frequency on losses". Journal of Turbomachinery, 138(11), p. 111006.

[10] Michelassi, V., Wissink, J. G., Frohlicj, J., and Rodi, W., 2003. "Large-eddy simulation of flow around low-pressure turbine blade with incoming wakes". AIAA Journal, 41, pp. 2143-2156.

[11] Matsuura, K., and Kato, C., 2007. "Large eddy simulation of compressible transitional flows in a low-pressure turbine cascade". AIAA Journal, 45, pp. 442-457.

[12] Medic, G., and Sharma, O., 2012. "Large-eddy simulation of flow in a low-pressure turbine cascade". ASME Turbo Expo Paper GT2012-68878.

[13] Wang, Y., Liu, H., Song, Y., and Chen, F., 2015. "Large eddy simulation of boundary layer separation and reattachment in a LPT blade at different incidence angles". ASME Turbo Expo Paper GT2015-42264.

[14] Jee, S., Joo, J., and Medic, G., 2016. "Large-eddy simulation of a high-pressure turbine vane with inlet turbulence". ASME Turbo Expo Paper GT2016-56980.

[15] Joo, J., Medic, G., and Sharma, O., 2016. "Large-eddy simulation investigation of impact of roughness on flow in a low-pressure turbine". ASME Turbo Expo Paper GT201657912.

[16] Joo, J., Emroy, M., Bose, S. T., Medic, G., Ham, F., and Sharma, O., 2016. "Large-eddy simulation of NACA65 compressor cascade with roughness". Proceedings of the Center for Turbulence Research summer program, pp. 343352.

[17] F.R. Menter, M. Kuntz, R. L., 2003. “Ten years of industrial experience with the SST turbulence model.”. In Turbulence, Heat and Mass Transfer 4, M. T. K. Hanjalic, Y. Nagano, ed., pp. 625-632.

[18] T. Knopp, B. Eisfeld, J. B. C., 2009. "A new extension for $k-\omega$ turbulence models to account for wall roughness". International Journal of Heat and Fluid Flow, 30, pp. 5465.

[19] Bassi, F., Crivellini, A., Rebay, S., and Savini, M., 2005. "Discontinuous Galerkin solution of the Reynoldsaveraged Navier-Stokes and $k-\omega$ turbulence model equations". Computers and Fluids, 34, pp. 507-540.

[20] Corsini, A., Rispoli, F., and Santoriello, A., 2005. "A variational multiscale higher-order finite-element formulation for turbomachinery flow computations". Computer Methods in Applied Mechanics and Engineering, 194, pp. 47974823.

[21] Cherednichenko, S., Frey, C., and Ashcroft, G., 2012.
"On the application of the Discontinuous Galerkin method to turbomachinery flows". European Congress on Computational Methods in Applied Sciences and Engineering, pp. 2359-2375.

[22] Ghidoni, A., Colombo, A., and Rebay, S., 2013. "Simulation of the transitional flow in a low pressure gas turbine cascade with a high-order Discontinuous Galerkin method". Journal of Fluids Engineering, 135, p. 071101.

[23] Carton de Wiart, C., Hillewaert, K., and Geuzaine, P., 2012. "DNS of a low pressure turbine blade computed with a Discontinuous Galerkin method". ASME Turbo Expo Paper GT2012-68900.

[24] Hillewaert, K., Carton de Wiart, C., Verheylewegen, G., and Arts, T., 2014. "Assessment of a high-order Discontinuous-Galerkin method for the direct numerical simulation of transition at low Reynolds number in the T106C high-lift low pressure turbine cascade". ASME Turbo Expo Paper GT2014-26739.

[25] Carton de Wiart, C., Hillewaert, K., Lorriaux, E., and Verheylewegen, G., 2015. "Development of a Discontinuous Galerkin solver for high quality wall-resolved/modelled DNS and LES of practical turbomachinery flows on fully unstructured meshes". ASME Turbo Expo Paper GT201543428.

[26] Garai, A., Diosady, L. T., Murman, S. M., and Madavan, N. K., 2015. "DNS of flow in a low-pressure turbine cascade using a Discontinuous-Galerkin spectral-element method". ASME Turbo Expo Paper GT2015-42773.

[27] Garai, A., Diosady, L. T., Murman, S. M., and Madavan, N. K., 2016. "DNS of low-pressure turbine cascade flows with elevated inflow turbulence using a DiscontinuousGalerkin spectral element method". ASME Turbo Expo Paper GT2016-56700.

[28] Garai, A., Diosady, L. T., Murman, S. M., and Madavan, N. K., 2017. "Scale-resolving simulations of bypass transition in a high-pressure turbine cascade using a spectralelement Discontinuous-Galerkin method". Journal of Turbomachinery, 140(3), p. 031004.

[29] Sharma, O., 2011. "The role of physical and numerical experiments in the development of high performance axial flow turbines". IGTI Scholar Lecture GT2011-46886.

[30] Ismail, F., and Roe, P. L., 2009. "Affordable, entropyconsistent Euler flux functions II: entropy production at shocks". Journal of Computational Physics, 228, pp. 5410-5436.

[31] Bassi, F., and Rebay, S., 2000. Discontinuous Galerkin methods: theory, computation and applications. Springer, Berlin, Heidelberg, Germany, ch. GMRES Discontinuous Galerkin solution of the compressible Navier-Stokes equations, pp. 197-208.

[32] Diosady, L. T., and Murman, S. M., 2013. "Design of a variational multiscale method for turbulent compressible 
flows". AIAA Computational Fluid Dynamics Conference 2013-2870.

[33] Diosady, L. T., and Murman, S. M., 2014. "DNS of flows over periodic hills using a Discontinuous-Galerkin spectral-element method". AIAA Paper 2014-2784.

[34] Diosady, L. T., and Murman, S. M., 2015. "Higher-order methods for compressible turbulent flows using entropy variables". AIAA Paper 2015-0294.

[35] Diosady, L. T., and Murman, S. M., 2017. "Tensor-product preconditioners for higher-order space-time discontinuous galerkin methods". Journal of Computational Physics, 330, pp. 296-318.

[36] Hu, F. Q., Li, X. D., and Lin, D. K., 2008. "Absorbing boundary conditions for nonlinear Euler and Navier-Stokes equations based on the perfectly matched layer technique". Journal of Computational Physics, 227, pp. 4398-4424.

[37] Garai, A., Diosady, L. T., Murman, S. M., and Madavan, N. K., 2016. "Development of a perfectly matched layer technique for a Discontinuous-Galerkin spectral-method". AIAA Paper 2016-1338.

[38] Diosady, L. T., and Murman, S. M., 2018. "A linearelasticity solver for higher-order space-time mesh deformation". AIAA Paper accepted for publication.

[39] Jespersen, D., Pulliam, T., and Buning, P., 1997. "Recent enhancements to OVERFLOW". AIAA Paper 97-0644.

[40] Alam, M., and Sandham, N. D., 2000. "Direct numerical simulation of 'short' laminar separation bubbles with turbulent reattachment". Journal of Fluid Mechanics, 403, pp. 223-250.

[41] Spalart, P. R., and Strelets, M. K., 2000. "Mechanisms of transition and heat transfer in a separation bubble". Journal of Fluid Mechanics, 403, pp. 329-349.

[42] Marxen, O., Lang, M., and Rist, U., 2013. "Vortex formation and vortex breakup in a laminar separation bubble". Journal of Fluid Mechanics, 728, pp. 58-90.

[43] Hussein, H. J., and Martinuzzi, R. J., 1996. "Energy balance for turbulent flow around a surface mounted cube placed in a channel". Physics of Fluids, 8, pp. 764-780.

[44] Breuer, M., Peller, N., Rapp, C., and Manhart, M., 2009. "Flow over periodic hills - numerical and experimental study in a wide range of Reynolds numbers". Computers and Fluids, 38, pp. 433-457.

[45] Gatski, T. B., 1997. New tools in turbulence modelling. Springer-Verlag, Berlin, Heidelberg, Germany, ch. Modelling compressibility effects on turbulence.

[46] Blonigan, P. J., 2017. "Adjoint sensitivity analysis of chaotic dynamical systems with non-intrusive least squares shadowing". Journal of Computational Physics, 348, pp. 803-826.

[47] Ekelschot, D., Ceze, M., Garai, A., and Murman, S. M., 2018. "Robust metric aligned quad-dominant meshing using $L_{p}$ centroidal voronoi tessellation". AIAA Paper 2018- 